\title{
Smoothed particle hydrodynamics applied in fluid structure interactions
}

\author{
A. Barreiro, A. J. C. Crespo, J. M. Domínguez \\ \& M. Gómez-Gesteira \\ EPHYSLAB Environmental Physics Laboratory, \\ Universidade de Vigo, Spain
}

\begin{abstract}
The capabilities of SPH models to deal with complex scenarios involving real geometries and extreme events are shown for different applications. The model not only is able to capture the main features of complex flows, but also allows including objects that can move due to the force exerted by the fluid. This is of special interest to reproduce the flooding of coastal areas, where water can move cars, pieces of urban furniture or debris.

Keywords: SPH, CFD, Lagrangian method, coastal protection.
\end{abstract}

\section{Introduction}

Smoothed particle hydrodynamics (SPH) [1] has become increasingly popular during the last decade to study free-surface flows. The conceptual simplicity of these Lagrangian models lies in the fact that the fluid is decomposed into a series of points, the particles, which interact with each other according to the fluid conservation laws. An overview of recent improvements in the technique is presented in the special issue SPH for free-surface flows [2].

Here we will briefly describe some cases where models based on SPH technique can be of interest to reproduce the interaction between water and coastal structures. Numerical results in the manuscript were obtained with DualSPHysics. This code, which is a joint effort by researchers at the University of Vigo (Spain) and the University of Manchester (UK), is freely available at (http://dual.sphysics.org) and has been applied to the study of coastal processes [3-13]. 


\section{Theory}

\subsection{The SPH method}

The main features of the SPH method are described in [9, 14-18]. Any function $A(r)$ can be approximated by an integral interpolant:

$$
A(\boldsymbol{r})=\int A\left(\boldsymbol{r}^{\prime}\right) W\left(\boldsymbol{r}-\boldsymbol{r}^{\prime}, h\right) \mathrm{d} \boldsymbol{r}^{\prime}
$$

where $W\left(\boldsymbol{r}-\boldsymbol{r}^{\prime}, h\right)$ is the weighting function or kernel and $h$ is weighting function smoothing length. In discrete notation, this leads to the following approximation at point $a$,

$$
A(\boldsymbol{r})=\sum_{b} m_{b} \frac{A_{b}}{\rho_{b}} W\left(\boldsymbol{r}-\boldsymbol{r}_{b}, h\right)
$$

where the summation is over all the particles $b$ within the region of compact support of the kernel function. The mass and density are denoted by $m_{b}$ and $\rho_{b}$ respectively, being $V_{b}=m_{b} / \rho_{b}$ the volume of a particle, $\boldsymbol{r}=\boldsymbol{r}_{a}$ the position vector and $W_{a b}=W\left(\boldsymbol{r}_{a}-\boldsymbol{r}_{b}, h\right)$ the weighting function (kernel) that depends on the distance between particles $a$ and $b$.

The model depends on the choice of the weighting function, which should satisfy several conditions such as positivity, compact support, and normalization. It also must be monotonically decreasing with increasing distance from particle $a$ [15-17]. Kernels depend on the non-dimensional distance between particles given by $q=r / h$, where $r$ is the distance between particles $a$ and $b$. The parameter $h$ controls the size of the area around particle $a$ where the contribution from other particles cannot be neglected. Although different kernels have been used in the literature, here we will consider the so called cubic spline.

$$
W(r, h)=\alpha_{D}\left\{\begin{array}{cc}
1-\frac{3}{2} q^{2}+\frac{3}{4} q^{3} & 0 \leq q \leq 1 \\
\frac{1}{4}(2-q)^{3} & 1 \leq q \leq 2 \\
0 & q \geq 2
\end{array}\right.
$$

In addition, the tensile correction [19] must be activated since the first derivative of the kernel goes to zero with $q$.

\subsection{Momentum equation}

The momentum conservation equation in a continuum field is

$$
\frac{D \boldsymbol{v}}{D t}=-\frac{1}{\rho} \nabla P+\boldsymbol{g}+\boldsymbol{\Gamma}
$$


where $\Gamma$ is the dissipative terms and $\boldsymbol{g}=(0,0,-9.81) \mathrm{ms}^{-2}$ the gravitational acceleration. In spite of different options for dissipation can be used [20] here we will only consider the artificial viscosity proposed by Monaghan [15]. Thus, Eq. (4) can be written in SPH notation as

$$
\frac{d \boldsymbol{v}_{a}}{d t}=-\sum_{b} m_{b}\left(\frac{P_{b}}{\rho_{b}^{2}}+\frac{P_{a}}{\rho_{a}^{2}}+\Pi_{a b}\right) \nabla_{a} W_{a b}+\boldsymbol{g}
$$

where $P_{k}$ and $\rho_{k}$ are the pressure and density of particle $k(a$ or $b$ ). The viscosity term $\Pi_{a b}$ is given by

$$
\Pi_{a b}=\left\{\begin{array}{cc}
\frac{-\alpha \overline{C_{a b}}}{\overline{\rho_{a b}}} & \boldsymbol{v}_{a b} \cdot \boldsymbol{r}_{a b}<0 \\
0 & \boldsymbol{v}_{a b} \cdot \boldsymbol{r}_{a b}>0
\end{array}\right.
$$

where the difference in position and velocity between particles $a$ and $b$ is $\boldsymbol{r}_{a b}=\boldsymbol{r}_{a}-\boldsymbol{r}_{\mathrm{b}}, \boldsymbol{v}_{a b}=\boldsymbol{v}_{a}-\boldsymbol{v}_{b}, \mu_{a b}=h \boldsymbol{v}_{a b} \cdot \boldsymbol{r}_{a b} /\left(r_{a b}^{2}+\eta^{2}\right)$, being $\overline{c_{a b}}=0.5\left(c_{a}+c_{b}\right)$ the mean speed of sound, $\eta^{2}=0.01 h^{2}$ and $\alpha$ a free parameter that depends on the problem.

\subsection{Continuity equation}

Changes in the fluid density are calculated in SPH using

$$
\frac{\mathrm{d} \rho_{a}}{\mathrm{~d} t}=\sum_{b} m_{b} \boldsymbol{v}_{a b} \nabla_{a} W_{a b}
$$

instead of using a weighted summation of mass terms as described in Monaghan [15], in order to prevent artificial density decrease near fluid interfaces.

\subsection{Equation of state}

The fluid in the SPH formalism is treated as weakly compressible and an equation of state is used to determine fluid pressure [21]. The compressibility is adjusted to slow the speed of sound so that the time step in the model (using a Courant condition based the speed of sound) is reasonable. Another limitation on compressibility is to restrict the sound speed to be at least ten times faster than the maximum fluid velocity, thereby keeping density variations to within less than $1 \%$.

Following [22, 23], the relationship between pressure and density follows the expression

$$
P=B\left[\left(\frac{\rho}{\rho_{0}}\right)^{\gamma}-1\right]
$$


where $\gamma=7$ and $B=c_{0}^{2} \rho_{0} / \gamma$ being $\rho_{0}=1000 \mathrm{~kg} \mathrm{~m}^{-3}$ the reference density and $c_{o}=c\left(\rho_{o}\right)=\left.\sqrt{(\partial P / \partial \rho)}\right|_{\rho_{o}}$ the speed of sound at the reference density.

\subsection{Moving the particles}

Particles move with a velocity close to the mean velocity in their neighborhood using the XSPH variant [24]

$$
\frac{d \boldsymbol{r}_{a}}{d t}=\boldsymbol{v}_{a}+\varepsilon \sum_{b} \frac{m_{b}}{\overline{\rho_{a b}}} \boldsymbol{v}_{b a} W_{a b}
$$

where $\varepsilon=0.5$ and $\overline{\rho_{a b}}=0.5\left(\rho_{a}+\rho_{b}\right)$.

\subsection{Density reinitialization}

In $\mathrm{SPH}$, the pressure field of the particles exhibits large pressure oscillations due to acoustic waves present in compressible fluids. One of the most straightforward and computationally least expensive methods to overcome this problem is to spatially filter the particles densities [25]. Although, there are several orders of correction available in DualSPHysics [20], here we will consider the zeroth- order correction, also called Shepard Filter, that must be applied every $\mathrm{M}$ time steps ( $\mathrm{M} \sim 30)$

$$
\rho_{a}^{\text {new }}=\sum_{b} \rho_{b} \tilde{W}_{a b} \frac{m_{b}}{\rho_{b}}=\sum_{b} m_{b} \tilde{W}_{a b}
$$

where the kernel has been corrected using a zeroth-order correction:

$$
\tilde{W}_{a b}=\frac{W_{a b}}{\sum_{b} W_{a b} \frac{m_{b}}{\rho_{b}}}
$$

\subsection{Boundary conditions}

In SPH, the boundary can be described by a set of discrete boundary particles which exert a repulsive force on fluid particles when they approach. Here, the Dynamic Boundary Conditions [3] will be used.

\subsection{Fluid-driven objects}

The movement of objects can be computed by summing the contributions exerted on the boundary particles for an entire body. Assuming that the objects are treated as rigid bodies, the force on each boundary particle is computed by 
summing up the contribution from the surrounding water particles. Hence, boundary particle $k$ experiences a force per unit mass given by

$$
\boldsymbol{f}_{k}=\sum_{a \in W P s} \boldsymbol{f}_{k a}
$$

where $\boldsymbol{f}_{k a}$ is the force per unit mass exerted by water particle $a$ on boundary particle $k$. The force exerted by a water particle on each boundary particle is given by

$$
m_{k} \boldsymbol{f}_{k a}=-m_{a} \boldsymbol{f}_{a k}
$$

invoking the principle of equal and opposite action and reaction.

For the motion of the moving body, the basic equations of rigid body dynamics are used

$$
\begin{gathered}
M \frac{d \boldsymbol{V}}{d t}=\sum_{k \in B P s} m_{k} \boldsymbol{f}_{k} \\
I \frac{d \boldsymbol{\Omega}}{d t}=\sum_{k \in B P s} m_{k}\left(\boldsymbol{r}_{k}-\boldsymbol{R}_{0}\right) \times \boldsymbol{f}_{k}
\end{gathered}
$$

where $M$ is the mass of the object, $I$ is the moment of inertia, $\boldsymbol{V}$ is the velocity of the object, $\boldsymbol{\Omega}$ its rotational velocity, $\boldsymbol{R}_{0}$ the position of the center of mass. Equations 14ab are integrated in time to predict the values of $\boldsymbol{V}$ and $\boldsymbol{\Omega}$ for the beginning of the next time-step. The velocity of each boundary particle that describes the moving body is then given by

$$
\boldsymbol{u}_{k}=\boldsymbol{V}+\boldsymbol{\Omega} \times\left(\boldsymbol{r}_{k}-\boldsymbol{R}_{0}\right)
$$

The boundary particles within the rigid body are moved by integrating Eq. (15) in time. This technique conserves both linear and angular momenta $[18,26]$.

\section{Study cases involving fluid structure interaction}

\subsection{Collision between waves and offshore structures}

A typical use of SPH models is the description of the violent collisions between freak waves and offshore structures. Figure 1 shows different instants of the collision between a large wave and an oil rig. This simulation which involves more than $10^{9}$ particles is, as far as we know, the largest simulation in computational fluid dynamics. The flow around the object is highly irregular and discontinuous. Thus, parcels of fluid are detached from the main bulk of water, evolve and blend again with the rest of the fluid. 


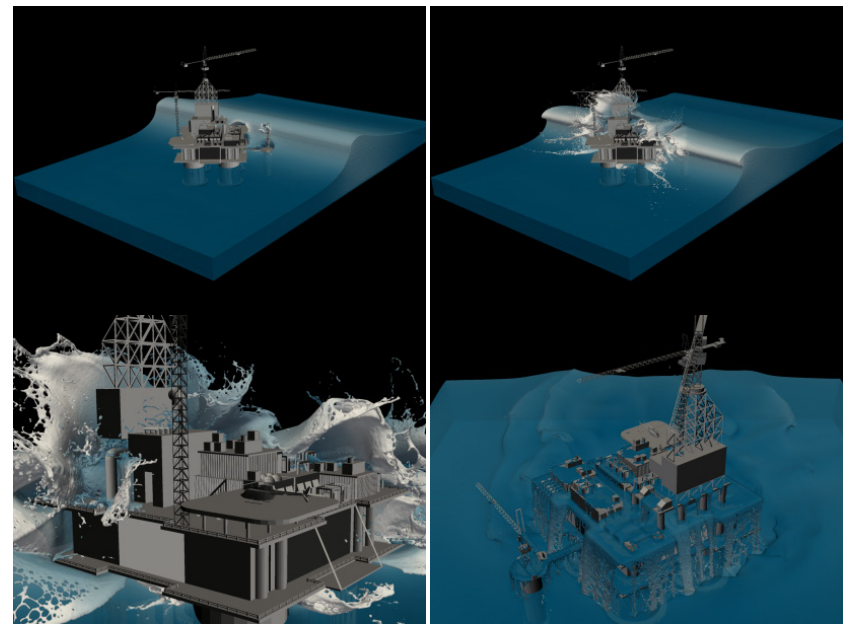

Figure 1: Collision between a solitary freak wave and an oil rig. The simulation involves more than $10^{9}$ particles.

\subsection{Design of breakwaters}

Due to the Lagrangian nature of SPH, these models have become a suitable tool to analyse breakwater. We can consider, for example, the run-up for a rubble mound breakwater, where the seaward layer is made of grooved blocks. The complex geometry of the blocks and the surrounding gaps within the breakwater seaward layer can be efficiently simulated to obtain a detailed description of the flow in order to analyze its response under wave attack. Run-up heights can be computed and compared with empirical solutions and experimental data for different wave conditions. Figure 2 shows the interaction of a wave with coastal defenses with different configurations.

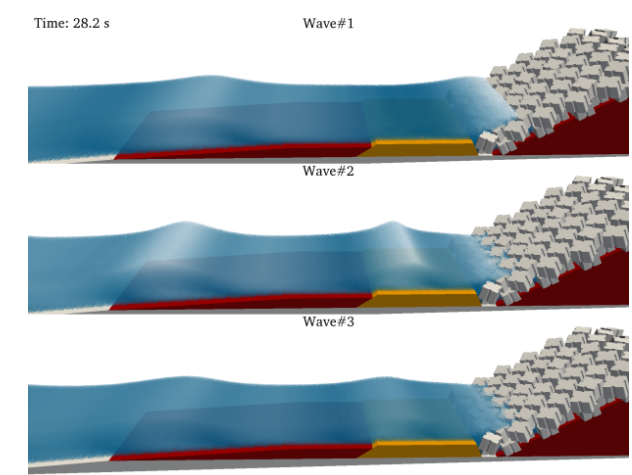

Figure 2: $\quad$ Interaction of a wave with different coastal structures. 
As mentioned above, the movement of the fluid through the voids and cavities among blocks is a key factor that determines the run-up and, hence, the efficiency of the defense. Figure 3 shows the movement of the fluid inside those cavities. Colors correspond to different instants, in such a way that the trajectory described by a set of particles can be followed.

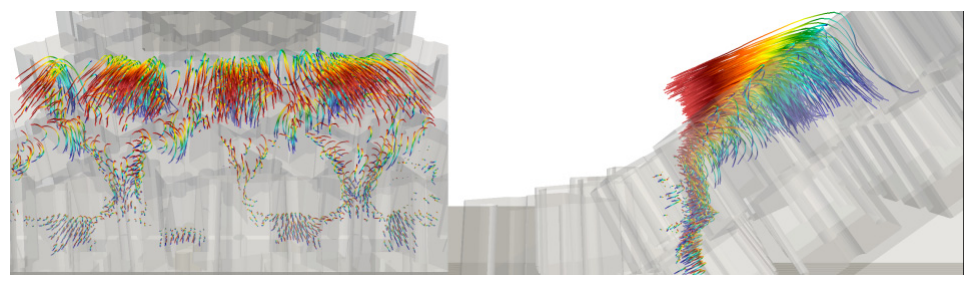

Figure 3: Trajectories of the particles through the interstices among the blocks for a structure with antifer configuration.

\subsection{Coastal flooding and damage on structures}

The model can be also used to analyse coastal flooding under extreme waves. Figure 4 shows how a large wave overtops the defenses of a small harbour and floods the adjacent area. Both the dimensions and the geometry of the area mimic a harbour located at the northern coast of the Iberian Peninsula. There, the model can be used for several purposes like calculating the maximum height attained by waves on the promenade or the forces exerted onto coastal objects. Figure 5 shows the collision of different waves with a lamppost. As the boundaries and objects are also formed by particles, the force on the structure is the summation of forces exerted on every particle.

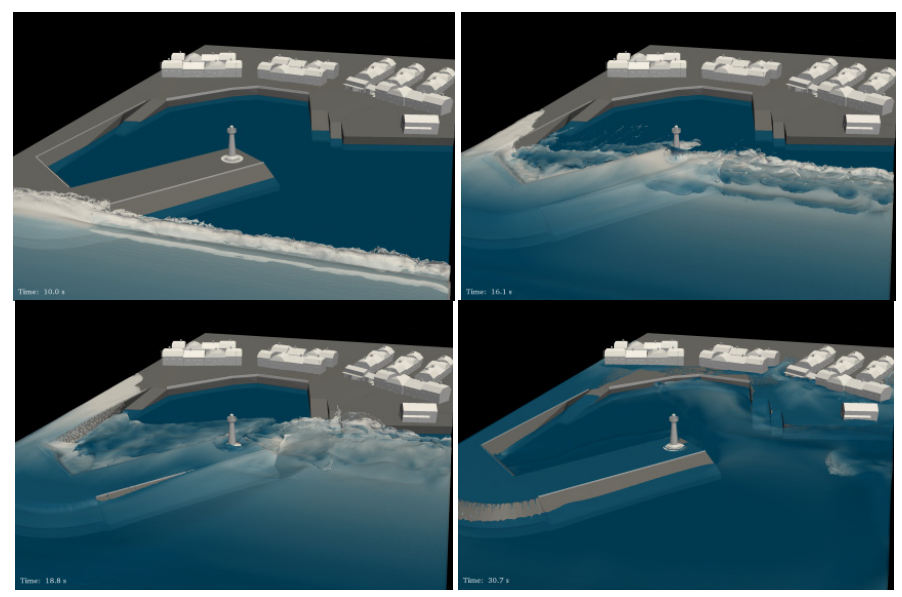

Figure 4: Flood by a large wave. Configuration similar to the harbour of O Barqueiro NW Spain. 

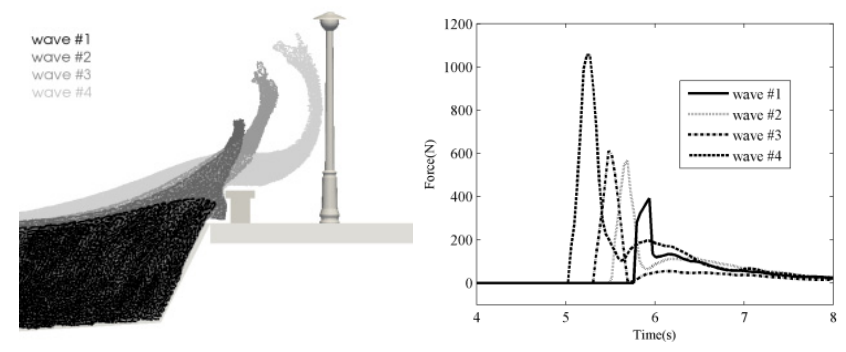

Figure 5: (a) Collision of different waves with a balustrade and a lamppost. (b) Maximum force exerted on the lamppost.

\subsection{Movement of objects}

Objects can also move under the force exerted by the fluid. This is of special interest when coastal areas are flooded and objects like cars, urban furniture or debris are dragged by the force of the fluid. Figure 6 shows the flood of a parking garage and how vehicles are dragged by the water.

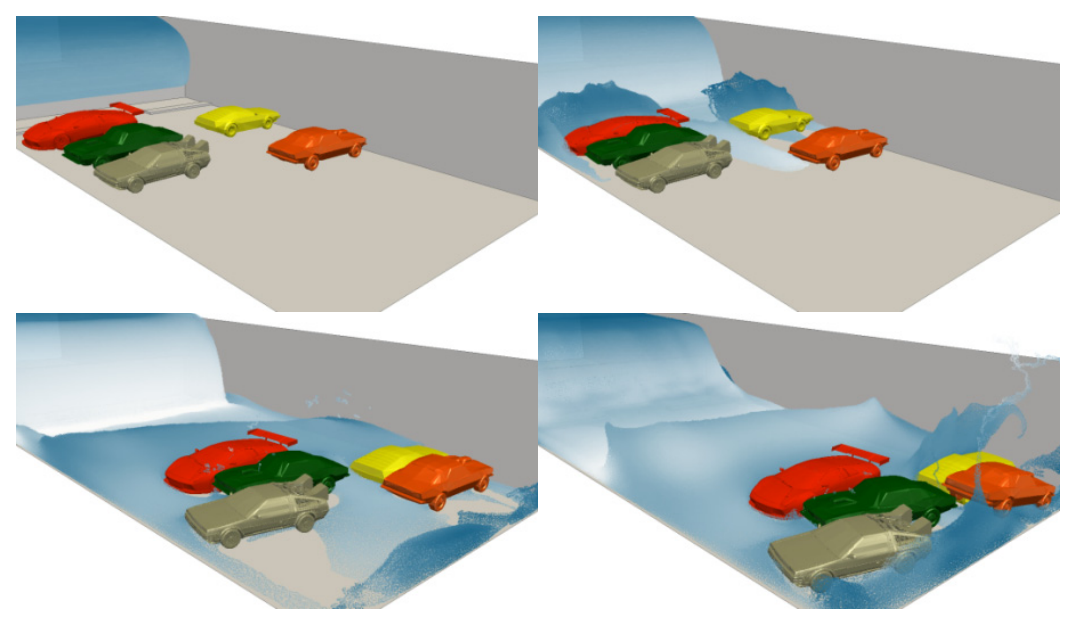

Figure 6: Flood of a parking area. Vehicles are washed away and collide with each other.

\section{Conclusion}

Here, we have shown the capabilities of the SPH models to deal with complex scenarios involving real geometries and extreme events. The Lagrangian nature of the method allows integrating the equations based on conservation (mass and momentum) without the constraints of a grid.

The model not only is able to capture the main features of complex flows, but also allows including objects that can move due to the force exerted by the fluid. 
This is of special interest to reproduce the flooding of coastal areas, where water can move cars, pieces of urban furniture or debris.

Finally, we should note that the model requires a high number of particles to generate accurate results, and the number of interactions among particles is considerably higher than in Eulerian methods. Thus, the computational requirements of these methods are huge for a single processor. This can be palliated with the use of parallelism. DualSPHysics is especially designed to take advantage of all resources of the computer. The model, can use in parallel different processing devices, both CPUs and GPUs, to speed up calculations.

\section{Acknowledgements}

This work was supported by the Universidade de Vigo under project INOU12-03 and also financed by the Ministerio de Economía y Competitividad under Project BIA2012-38676-C03-03. We want to acknowledge Barcelona Supercomputer Center (BSC-CNS) for their support and the use of their resources under the current activity FI-2012-3-0004: Massively Parallel Smoothed Particle Hydrodynamics scheme using GPU clusters.

\section{References}

[1] Gingold, R.A., Monaghan, J.J., 1977. Smoothed particle hydrodynamics: Theory and application to non-spherical stars. Monthly Notices of the Royal Astronomical Society 181, 375-389.

[2] Gómez-Gesteira, M., Rogers, B.D., Violeau, D., Grassa, J.M., Crespo, A.J.C., 2010a. SPH for free-surface flows. Journal of Hydraulic Research 48, 3-5. doi: 10.3826/jhr.2010.0014.

[3] Crespo, A.J.C., Gómez-Gesteira, M., Dalrymple, R.A., 2007a. Boundary conditions generated by dynamic particles in SPH methods. Computers, Materials \& Continua, 5, 173-184.

[4] Crespo, A.J.C., Gómez-Gesteira, M., Dalrymple, R.A. 2007b. 3D SPH Simulation of large waves mitigation with a dike. Journal of Hydraulic Research 45, 631-642.

[5] Crespo, A.J.C., Gómez-Gesteira, M., Dalrymple, R.A., 2008. Modeling dam break behavior over a wet bed by a SPH technique. Journal of Waterway, Port, Coastal and Ocean Engineering 134, 313-320.

[6] Dalrymple, R.A., Rogers, B.D., 2006. Numerical modeling of water waves with the SPH method. Coastal Engineering 53, 141-147.

[7] Gómez-Gesteira, M., Dalrymple, R.A., 2004. Using a 3D SPH method for wave impact on a tall structure. Journal of Waterway, Port, Coastal and Ocean Engineering 130, 63-69.

[8] Gómez-Gesteira, M., Cerqueiro, D., Crespo, C., Dalrymple, R.A., 2005. Green water overtopping analyzed with a SPH model. Ocean Engineering 32, 223-238. 
[9] Gómez-Gesteira, M., Rogers, B.D., Dalrymple, R.A., Crespo, A.J.C., 2010b. State-of-the-art of classical SPH for free-surface flows. Journal of Hydraulic Research 48, 6-27. doi: 10.3826/jhr.2010.0012.

[10] Narayanaswamy, M.S., Crespo, A.J.C., Gómez-Gesteira, M., Dalrymple, R.A., 2010. SPHysics-Funwave hybrid model for coastal wave propagation. Journal of Hydraulic Research 48, 85-93. doi: 10.3826/jhr.2010.0007.

[11] Rogers, B.D., Dalrymple, R.A., 2008. SPH Modeling of tsunami waves, Advances in Coastal and Ocean Engineering, Vol. 10. In Advanced Numerical Models for tsunami waves and runup. World Scientific.

[12] Rogers, B.D., Dalrymple, R.A., Stansby, P.K., 2010. Simulation of caisson breakwater movement using SPH. Journal of Hydraulic Research 48, 135141. doi: 10.3826/jhr.2010.0013.

[13] Omidvar, P., Stansby, P.K., Rogers, B.D., 2011. Wave body interaction in 2D using Smoothed Particle Hydrodynamics (SPH) with variable particle mass. International Journal for Numerical Methods in Fluids. doi: 10.1002/fld.2528.

[14] Monaghan, J.J., 1982. Why particle methods work. SIAM Journal on Scientific and Statistical Computing 3, 422-433.

[15] Monaghan, J.J., 1992. Smoothed particle hydrodynamics. Annual Review of Astronomy and Astrophysics 30, 543- 574.

[16] Benz, W., 1990.Smoothed Particle Hydrodynamics: A review. In The numerical Modelling of Nonlinear Stellar Pulsations: Problems and Prospects, J.R. Butchler ed., Kluwer Academic Publ. 269-288.

[17] Liu, G.R., 2003. Mesh Free methods: Moving beyond the infinite element method, CRC Press.

[18] Monaghan, J.J., 2005. Smoothed Particle Hydrodynamics. Reports on Progress in Physics 68, 1703-1759.

[19] Monaghan, J.J., 2000. SPH without tensile instability. Journal of Computational Physics 159, 290-311.

[20] Gómez-Gesteira, M., Rogers, B.D., Crespo, A.J.C., Dalrymple, R.A., Narayanaswamy, M., Domínguez, J.M., 2012a. SPHysics - development of a free-surface fluid solver- Part 1: Theory and Formulations. Computers and Geosciences. 48 289-299.

[21] Monaghan, J.J., 1994. Simulating free surface flows with SPH. Journal of Computational Physics 110, 399- 406.

[22] Monaghan, J.J., Kos, A., 1999. Solitary waves on a Cretan beach. Journal of Waterway, Port, Coastal and Ocean Engineering 125, 145-154.

[23] Batchelor, G.K., 1974. Introduction to fluid dynamics, Cambridge University Press.

[24] Monaghan, J.J., 1989. On the problem of penetration in particle methods. Journal of Computational Physics 82, 1-15.

[25] Colagrossi A., Landrini, M., 2003. Numerical simulation of interfacial flows by smoothed particle hydrodynamics. Journal of Computational Physics 191, 448-475.

[26] Monaghan, J.J., Kos, A., Issa, N., 2003. Fluid motion generated by impact. Journal of Waterway, Port, Coastal and Ocean Engineering 129, 250-259. 